\title{
Coherence Switching with Metamaterials
}

\author{
Matias Koivurova $\odot,{ }^{1,2, *}$ Tommi K. Hakala, ${ }^{3}$ Jari Turunen, ${ }^{3}$ Ari T. Friberg, ${ }^{3}$ \\ Humeyra Caglayan ${ }^{1},{ }^{1, \dagger}$ and Marco Ornigotti ${ }^{1, \$}$ \\ ${ }^{1}$ Tampere University, Faculty of Engineering and Natural Sciences, 33720 Tampere, Finland \\ ${ }^{2}$ Tampere University, Tampere Institute for Advanced Study, 33100 Tampere, Finland \\ ${ }^{3}$ Institute of Photonics, University of Eastern Finland, P.O. Box 111, FI-80101 Joensuu, Finland
}

(Received 7 May 2021; accepted 13 September 2021; published 6 October 2021)

\begin{abstract}
We demonstrate, theoretically, how the insertion of an enhanced epsilon-near-zero (EENZ) mirror in a laser cavity grants exceptional control over the coherence properties of the emitted light beam. By exploiting the peculiar sensitivity to polarization of EENZ materials, we achieve superior control over the spatial coherence of the emitted laser light, which can be switched at will between nearly incoherent and fully coherent, solely by means of polarization optics. Our EENZ cavity design is expected to be an efficient, compact, reconfigurable, and easily scalable source of light for illumination and speckle contrast imaging, as well as any other application that benefits from controlled spatial coherence.
\end{abstract}

DOI: $10.1103 /$ PhysRevLett.127.153902

Coherence is one of the defining properties of light, and there exist many measurement modalities which take advantage of either the presence or absence of correlations. For example, lasers are usually highly coherent sources, which have many useful properties. One such property is the unmatched brightness of coherent laser light, being desirable in most imaging applications. However, the formation of speckle is a central limiting factor in illumination. A speckle pattern is generated when a highly spatially coherent field is imposed with a rapidly varying phase across its wavefront. Speckle is commonly encountered, for example, when coherent light is reflected from an optically rough surface. If the light is of high coherence, the speckle contrast is also high, causing uneven illumination and a loss of information in the produced image [1]. Although it results in a lower brightness, the usual illumination approach is to employ light with a low degree of spatial coherence. The predominant method in microscopy is Köhler illumination [2], which allows for the uniform lighting of a sample.

On the other hand, speckle can be used for detecting and imaging flow. This method is called speckle contrast imaging $[3,4]$, and the central idea is to use coherent light to produce speckle from a time-varying sample. As a fluid flows, it will cause the wavefront of the light to attain a time-varying phase. Thus, where flow is present, each speckle pattern is different, and averaging over a long

Published by the American Physical Society under the terms of the Creative Commons Attribution 4.0 International license. Further distribution of this work must maintain attribution to the author(s) and the published article's title, journal citation, and DOI. enough detection time results in a clear image of flow areas. Köhler illumination and speckle contrast imaging are both useful in medicine and biology. Integrating these into a single system would be highly beneficial, allowing for rapid switching between traditional high resolution imaging and flow detection with speckle contrast. Novel laser design has been centered on generating a low degree of coherence for imaging [5-8], inertial confinement [9-11] and free-space telecommunication $[12,13]$ applications, to name a few. As an extension of these, light sources that feature switchable coherence properties [14-17] have gained considerable attention. Although they can achieve some degree of coherence switching, these methods employ relatively large $4 f$ imaging systems, which necessarily require mechanical parts.

In the present Letter, we introduce solid-state coherence switching based on enhanced epsilon-near-zero (EENZ) material, on which we recently reported in Ref. [18]. The EENZ consists of two different types of parallel planar layers of homogeneous media, where one is a dielectric, and the other one an epsilon-near-zero (ENZ) material. The EENZ structure amplifies the refractive and/or reflective properties of the constituent ENZ, and here we present a possible experimental scheme for coherence filtering and switching, which is achieved by replacing a normal mirror with an EENZ mirror inside a laser cavity. Coherence control is achieved by switching between transverse electric (TE) and transverse magnetic (TM) polarization, as the employed metamaterial produces largely different responses to the different polarizations. This is mainly due to the plasmon excitations inside the structure, which only exist for TM polarization. When plasmonic modes are present, the structure causes significant losses for light that is not at normal incidence to the EENZ surface. To analytically model these types of structures, we use the 


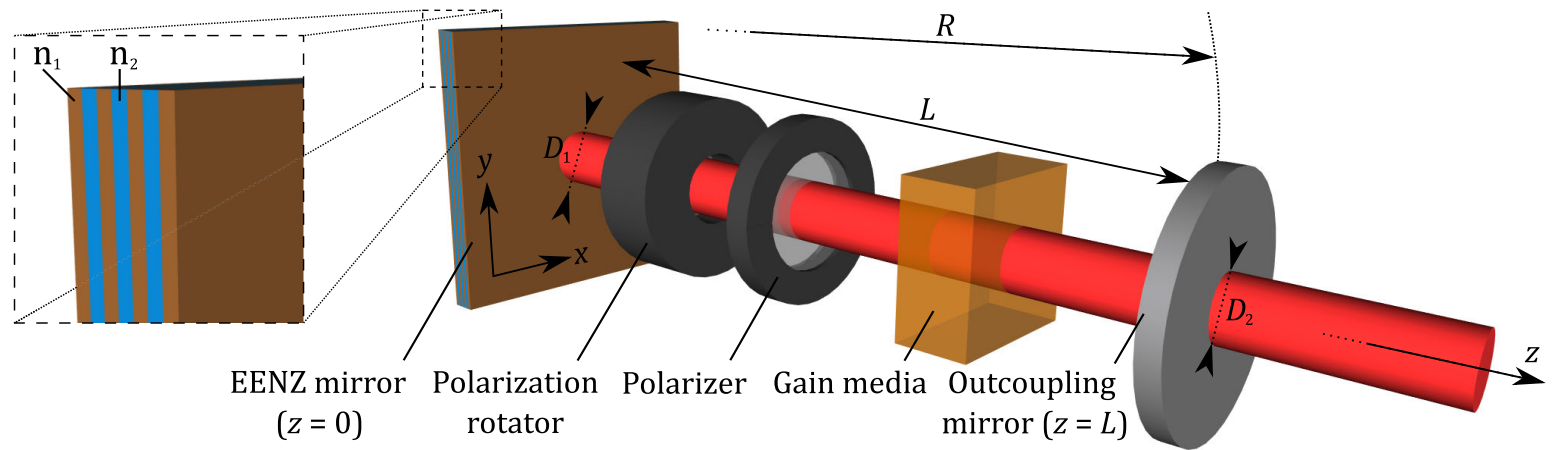

FIG. 1. Schematic of the proposed near plane parallel laser cavity, inset shows EENZ mirror structure. The device consists of an EENZ back mirror ( $n_{1}$ and $n_{2}$ being the refractive indices and $D_{1}$ the effective aperture), a polarization rotator, a linear polarizer, gain media, and an outcoupling mirror (with aperture $D_{2}$ and radius of curvature $R$ ). The length of the cavity is $L$.

theory of stratified media, which is considered in detail in Ref. [18]. We find that very strong coherence switching is possible, going from nearly incoherent to completely coherent with no mechanical parts.

Laser cavities often support mutually uncorrelated Hermite-Gaussian (HG) modes [19], and the number of these transverse modes depends on the geometry of the end mirrors [20]. Therefore, the main challenge for coherence control is the ability to reduce the number of oscillating modes at will, without changing the cavity geometry. We show that this is possible to achieve with the setup depicted in Fig. 1, where a schematic of the EENZ material (inset) is shown together with the proposed experimental setup. To demonstrate how the EENZ can drastically impact coherence control inside the cavity depicted in Fig. 1, let us assume that the light emitted from the gain material first propagates through the linear polarizer oriented along the $x$ axis, and the polarization rotator, which rotates the polarization counterclockwise to angle $\phi$ with respect to the $x$ axis. It then reflects from the back mirror, and thus, is filtered by the reflection coefficient of the EENZ, denoted by

$$
\mathbf{r}_{e}\left(k_{x}\right)=\left[\begin{array}{cc}
r_{\mathrm{TE}}\left(k_{x}\right) & 0 \\
0 & r_{\mathrm{TM}}\left(k_{x}\right)
\end{array}\right],
$$

where $r_{\mathrm{TE}}$ and $r_{\mathrm{TM}}$ are complex-valued functions of the spatial frequency $k_{x}$. Moreover, we have implicitly assumed the fields in the cavity to be quasimonochromatic, and purposely omitted the $\omega$ dependence for brevity, since frequency dispersion was found to be negligible. The light continues to propagate and goes through the polarization optics again, finally meeting the outcoupling mirror with reflection coefficient $r$, and going back to the gain material, completing a single round-trip. Afterward, the intracavity field has the form

$$
E\left(k_{x}\right)=E_{0}\left(k_{x}\right) r\left[r_{\mathrm{TE}}\left(k_{x}\right) \cos ^{2} \phi-r_{\mathrm{TM}}\left(k_{x}\right) \sin ^{2} \phi\right],
$$

and a detailed derivation of the equation is found in Supplemental Material [21]. From the relation above we see that by simply choosing the polarization rotation angle $\phi$ properly, it is possible switch between TE and TM modulations.

For a functioning laser cavity, it is vital to have as low losses as possible, and only the modes which have larger round-trip gain than loss will contribute to lasing. With this in mind, we designed a highly reflecting EENZ mirror with a maximum reflection of $\left|r_{\mathrm{TM}}\right|^{2} \sim 96.8 \%$ at the operating wavelength of $1422 \mathrm{~nm}$. Note that the choice of operating wavelength is arbitrary, and the structure can be designed for any wavelength where there are suitable materials. The EENZ mirror consists of 65 ENZ and 64 dielectric layers, starting and ending with ENZ, with layer thicknesses of 64 and $225 \mathrm{~nm}$ for the ENZ and dielectric, respectively. With this large number of layers, we seek to find the theoretical maximum performance of EENZ, and a smaller number of layers is still feasible for this application [18]. We chose indium tin oxide as the ENZ with $n_{1}=\sqrt{i 0.6}$, and titanium dioxide as the dielectric with $n_{2}=2.5$, as they are readily available materials for possible experimental verification (see Ref. [18] for more details).

The computed reflectance is shown in Fig. 2, from which it is evident that the structure has a strong angle and polarization dependent response. The maximum reflection for TM-polarized light occurs at exactly normal incidence, whereas the TE polarization has a broad maximum. Therefore, we can choose our gain such that the roundtrip losses equal the round-trip gain only for normal incidence when the rotator angle is set to $\phi=\pi / 2$. This will force single mode operation, regardless of the cavity configuration (given that the resonator is stable). If the angle is set to $\phi=0$, the usual multimode action will resume.

Assuming that the outcoupling mirror has a high reflectivity of $|r|^{2}=0.995$ and other cavity losses amount to approximately $\gamma_{i} \sim 0.05$ per round-trip, we find that the total loss for light at normal incidence to the EENZ is

$$
\gamma=-\ln \left(|r|^{2}\left|r_{\mathrm{TM}}\right|^{2}\right) / 2-\ln \left(1-\gamma_{i}\right) \approx 0.07,
$$




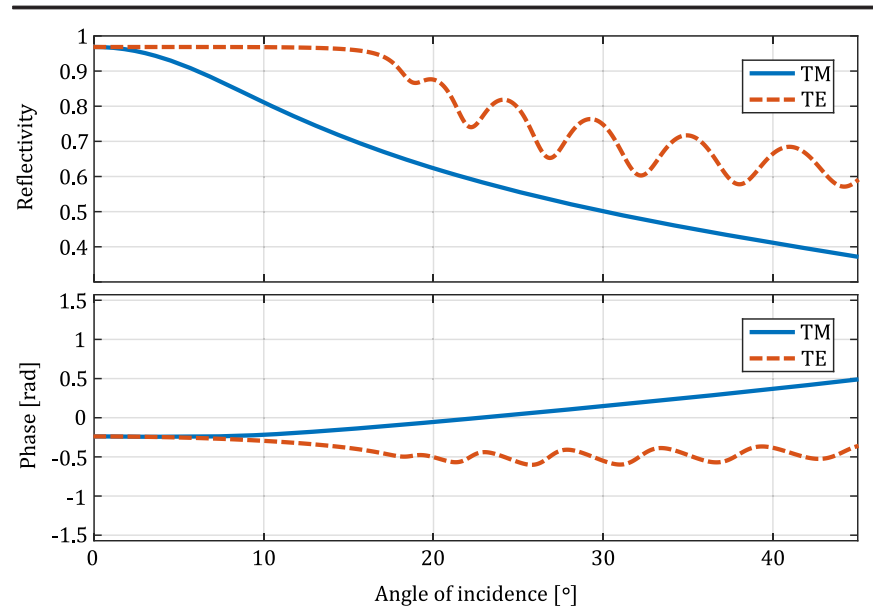

FIG. 2. Top: angular reflectivity and bottom: phase of the EENZ cavity mirror for TM and TE polarizations. Reflectivity of the TE response remains above the maximum TM reflectivity of $96.8 \%$ over a 6.45 degree angle (a considerably enlarged version is found in Supplemental Material [21]).

and the gain coefficient $G$ needs to be of at least equal magnitude. A good candidate for a gain material in an experimental setting would be chromium doped yttrium aluminium garnet $\left(\mathrm{Cr}^{4+}\right.$ :YAG) crystal, since its emission can be tuned to our design wavelength and YAG crystals commonly exceed the required gain coefficient of 0.07 .

However, since the reflection coefficient of the EENZ mirror imparts a small but finite phase to the electric field, we need to evaluate its effect on the modes oscillating within the cavity. To do this, we use an adaptation of the Fox-Li algorithm [22], which is analogous to the iterative Fourier transform algorithm commonly used in diffractive optics. The algorithm propagates the intracavity field between resonator mirrors at $z=0$ and $z=L$, with $L$ being the cavity length. The mirrors are treated as thin elements that truncate the field incident on them, and possibly add a phase term on reflection. We assume that the modes are separable, such that the field inside the cavity can be written as $\boldsymbol{\psi}_{m n}(x, y, L)=\hat{\mathbf{x}} \psi_{m}(x, L) \psi_{n}(y, L)$. Hence, we can carry out our analysis for the modes in the $x$ direction, and a similar procedure will hold for the modes in the $y$ direction.

To illustrate how this method works, let us first consider a cavity such as in Fig. 1, but with a regular planar mirror with aperture $D_{1}$, instead of the EENZ mirror placed in the plane $z=0$. Moreover, we take the second cavity mirror at $z=L$ to be spherical with a diameter $D_{2}$ and a radius of curvature $R$. To find a (scalar) mode $\psi(x)$, we may start from some initial guess for the field at $z=0$. The field is propagated to $z=L^{-}$just in front of mirror 2 using the angular spectrum approach

$$
\psi\left(x, L^{-}\right)=\int_{-\infty}^{\infty} a\left(k_{x}, 0\right) \exp \left[i\left(k_{x} x+k_{z} L\right)\right] d k_{x},
$$

where $k_{x}$ is the transverse wave vector component along the $x$ axis,

$$
a\left(k_{x}, 0\right)=\frac{1}{2 \pi} \int_{-D_{1} / 2}^{D_{1} / 2} \psi(x, 0) \exp \left(-i k_{x} x\right) d x
$$

is the angular spectrum, and $k_{z}=\sqrt{k_{0}^{2}-k_{x}^{2}}$, is the wave vector component toward the propagation direction.

It is convenient to "unfold" the resonator by considering it as a periodic system where mirrors are replaced with lenses. The lens at $z=L$ has a focal length $F$ and the field after it is

$$
\psi\left(x, L^{+}\right)=\psi\left(x, L^{-}\right) \exp \left(-i k_{0} x^{2} / 2 F\right) .
$$

This field is then propagated to mirror 1 with the angular spectrum method. Since mirror 1 is planar, we continue the propagation by just truncating the result to get $\psi\left(x, 2 L^{+}\right)$. This completes a single round-trip, and the next one can be started with the result of the first one. If we start the iteration with a smooth guess, we end up with the fundamental mode $\psi_{0}(x)$, i.e., $\mathrm{TEM}_{00}$. With more finely structured initial guesses the propagation will lead to higher-order modes $\psi_{m}(x)$.

The algorithm has to run until the results converge. After a sufficient number of round-trips $n$, we obtain a region where the condition

$$
\psi_{m}[x,(n+2) L]=\gamma_{m} \psi_{m}(x, n L)
$$

is satisfied. In this region we know the oscillating modes and also the factors $\gamma_{m}$, which give the round-trip intensity losses $\left|\gamma_{m}\right|^{2}$. Only those resonator modes for which the intensity gain $G$ exceeds the loss are above the lasing threshold, implying that we can calculate coefficients $c_{m}=G-\left|\gamma_{m}\right|^{2}$, which have to be positive for lasing to occur.

The resonator was designed such that (slightly perturbed) HG modes are generated, with the design details given in the Supplemental Material [21]. Assuming a waist of width $w_{0}$ at $z=0$, the beam width at $z=L$ is

$$
w=w_{0} \sqrt{1+\left(L / z_{R}\right)^{2}},
$$

whereas the radius of curvature of the field is equal to the curvature of the outcoupling mirror

$$
R=L+\frac{z_{R}^{2}}{L}
$$

where $z_{R}=\pi w_{0}^{2} / \lambda_{0}$ is the Rayleigh range. In the unfolded model considered above, we now choose the focal length as $F=R / 2$ so that the HG modes propagate periodically.

The iteration starts from the HG mode at $z=L^{-}$, with $w$ and $R$ given by Eqs. (8) and (9), so that within $|x|<D_{2} / 2$ we have 


$$
\begin{aligned}
\psi_{m}\left(x, L^{-}\right)= & \left(\frac{2}{\pi w^{2}}\right)^{1 / 4} \frac{1}{\sqrt{2^{m} m !}} H_{m}\left(\frac{\sqrt{2} x}{w}\right) \\
& \times \exp \left(-\frac{x^{2}}{w^{2}}\right) \exp \left[i \alpha_{m}(x)\right]
\end{aligned}
$$

with a phase term

$\alpha_{m}(x)=k_{0} L-(m+1 / 2) \arctan \left(L / z_{R}\right)+\frac{k_{0} x^{2}}{2 R}$,

and $k_{0}=2 \pi / \lambda_{0}$ is the central wave number.

Let us now replace mirror 1 with the EENZ mirror and add the polarization optics. To apply this method to our EENZ cavity, the standard Fox-Li algorithm needs to be modified for two reasons. First, we need to account for the polarization elements. Second, it is more natural to model the EENZ mirror in the space-frequency domain rather than in the spatial domain. Both of these are accomplished simply by writing the $x$-polarized mode in analogy with Eq. (2), which takes on the form

$a_{m}\left(k_{x}, 2 L^{+}\right)=a_{m}\left(k_{x}, 2 L^{-}\right)\left[r_{\mathrm{TE}} \cos ^{2} \phi-r_{\mathrm{TM}} \sin ^{2} \phi\right]$,

after interacting with the EENZ mirror. As there is no need to Fourier transform to the spatial domain, we can skip that step and continue propagation to mirror 2 .

Once the Fox-Li algorithm has been applied on all laser modes that exceed the lasing threshold, we form the crossspectral density (CSD) of the output field along the $x$ axis in the form of a Mercer-type expansion

$$
W\left(x_{1}, x_{2}\right)=\sum_{m=0}^{M} c_{m} \psi_{m}^{*}\left(x_{1}\right) \psi_{m}\left(x_{2}\right),
$$

where $c_{m}=G-\left|\gamma_{m}\right|^{2}$ are the mode weights discussed below Eq. (7), $\psi_{m}(x)$ refers to the converged Fox-Li mode having just exited the resonator, and $M$ is the highest-order mode that can lase. This expansion should not be confused with the rigorous coherent mode representation of the CSD [23], in which the coherent modes are eigenfunctions of the homogeneous Fredholm integral equation. In the nondegenerate case these modes are then precisely the laser cavity modes [24]. Rather, Eq. (13) is a phenomenological expansion, where the modes need not be necessarily orthogonal. A further point here is that since we consider narrow linewidth (quasimonochromatic) light, the various HG modes can be thought of as mutually uncorrelated on time averaging.

To attain a single numerical value for the coherence of the field, we employ the overall degree of coherence, as in

$$
\bar{\mu}^{2}=\frac{\iint_{-\infty}^{\infty}\left|W\left(x_{1}, x_{2}\right)\right|^{2} d x_{1} d x_{2}}{\iint_{-\infty}^{\infty} S\left(x_{1}\right) S\left(x_{2}\right) d x_{1} d x_{2}}
$$

where $S(x)=W(x, x)$ is the spatial intensity distribution. This quantity is bounded to the interval $[0,1]$, with 0 and 1 corresponding to complete incoherence and coherence, respectively.

Additionally, to obtain a more in-depth look at the coherence properties of the field, we record the full-width-at-half-maximum (FWHM) values for the intensity distribution and coherence width, denoted by $w$ and $\sigma$, respectively. We rotate the correlation function by changing to average and difference coordinates, $\bar{x}=\left(x_{1}+x_{2}\right) / 2$, and $\Delta x=x_{2}-x_{1}$, where we find the intensity width along the mean coordinate and the coherence width along the difference. The intensity width is constant, but the coherence width can vary across the beam, i.e., $\sigma(\bar{x})$ is not constant, and therefore we compute the intensity weighted coherence width as in

$$
\sigma=\frac{\int_{-\infty}^{\infty} \sigma(\bar{x}) S(\bar{x}) d \bar{x}}{\int_{-\infty}^{\infty} S(\bar{x}) d \bar{x}}
$$

For our simulations, we employed the following cavity parameters: $L=100 \mathrm{~mm}, R=10 L, D_{1} \gg D_{2}$, $D_{2}=7.5 \mathrm{~mm}$. These choices lead to a stable cavity, supporting up to $43 \mathrm{HG}$ modes. Although this is entirely adequate for our purposes, it is feasible to design cavities which support even more modes, if necessary. The computed overall degree of coherence for both TM and TE responses as a function of pump power is shown in Fig. 3. It was found that the TE response of the EENZ mirror only slightly perturbed the modes within the cavity, and had no significant effect on the overall degree of coherence, which attained a minimum of $\bar{\mu}=0.153$ with strong pumping, corresponding to $w=5.8 \mathrm{~mm}$ and $\sigma=88.4 \mu \mathrm{m}$. Moreover, the TM response allowed the oscillation of only the fundamental mode at threshold, thus producing completely coherent light with $\bar{\mu}=1$ and $w=\sigma=615 \mu \mathrm{m}$.

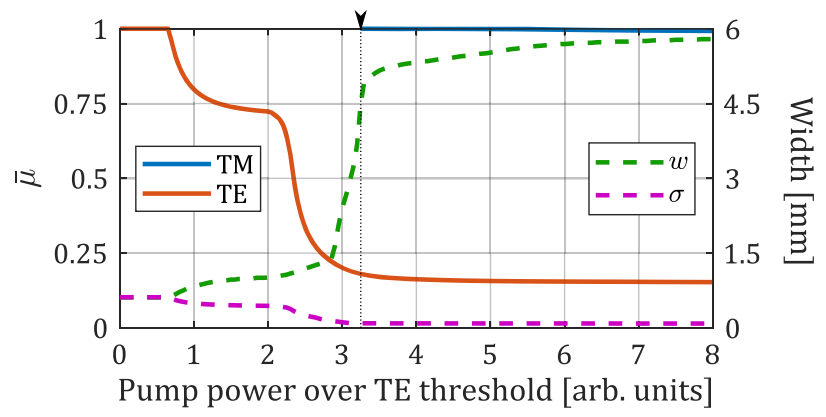

FIG. 3. Overall degree of coherence $\bar{\mu}$ as a function of increasing pump power, starting from the TE threshold (with $\phi=0)$. The TM mode $(\phi=\pi / 2)$ has a higher lasing threshold (denoted by the arrow) due to the finite width of the angular spectrum of the fundamental mode. The intensity and coherence widths, $w$ and $\sigma$, are shown for the TE response. In the case of $\mathrm{TM}$, these two quantities remained constant, and equal to the TE case near threshold. 
The TM response has a higher threshold than the TE (highlighted with an arrow in Fig. 3), since not even the fundamental mode could oscillate at the lower pumping levels. This is because the angular spectrum of the fundamental mode is wider than the maximum of the TM reflectivity at lower pumping levels; once the threshold is reached, only the fundamental mode oscillates. Moreover, as the pumping is increased, the overall degree of coherence remains very close to unity, since the TM response actively modifies the angular spectrum of the oscillating mode. In our simulations, it was found that every higher-order mode is slowly modified to correspond to the fundamental mode as the intracavity field evolves. However, this evolution was relatively slow, and a full 1 000000 round-trips was required for the results to approximately converge (corresponding to $333.6 \mu \mathrm{s}$ of time evolution). For a mirror with lower ENZ losses, the reflectivity becomes more narrow, and the modes converge faster. A detailed discussion on the convergence behavior of our algorithm can be found in Supplemental Material [21], and the generated CSDs are presented as a function of increasing pump power in Supplemental Material videos.

In contrast, the TE response could oscillate starting from the threshold discussed in connection with Eq. (3), due to the remarkable flatness of the TE response. Additionally, since the deviation from a normal mirror was so small, the HG modes did not need an excessive amount of round-trips for the result to converge. On the contrary, too many roundtrips caused numerical instabilities to blow up, since the TE response of the EENZ mirror has minor off-axis peaks. Therefore, for the TE modes, we propagated the intracavity field for 1000 round-trips. The only difference to a normal laser cavity was that the HG modes were about $5 \%$ wider.

In the present Letter, we have demonstrated solid-state coherence switching with polarization optics and an EENZ mirror. The low coherence state depends on the number of oscillating modes, thus relying on the cavity geometry. On the other hand, the high coherence state is generated by increasing the losses for light that is not propagating along the normal of the EENZ mirror plane. Consequently, the system can achieve both single-mode operation and complete spatial coherence regardless of the laser resonator geometry. Similar results may be attained with other polarization dependent elements. It is important to point out that although the analysis carried out within this work is entirely theoretical, the proposed set of parameters and specific material values have been carefully chosen to allow an experimental verification of the coherence switching induced by the EENZ mirror. The performance of the EENZ mirror depends only on the losses of the constituting ENZ layers, and lower losses will improve the operation of the device.

The authors acknowledge the financial support of the Academy of Finland Flagship Programme
(PREIN-decisions 320165, 320166). H. C. acknowledges financial support of the European Research Council (Starting Grant project aQUARiUM; Agreement No. 802986), T. K. H. acknowledges Academy of Finland Project No. 322002, and A. T. F. acknowledges Academy of Finland Project No. 310511.

*matias.koivurova@tuni.fi

†humeyra.caglayan@tuni.fi

*marco.ornigotti@tuni.fi

[1] J. M. Artigas, A. Felipe, and M. J. Buades, Contrast sensitivity of the visual system in speckle imagery, J. Opt. Soc. Am. A 11, 2345 (1994).

[2] M. Saxena, G. Eluru, and S. S. Gorthi, Structured illumination microscopy, Adv. Opt. Photonics 7, 241 (2015).

[3] D. A. Boas and A. K. Dunn, Laser speckle contrast imaging in biomedical optics, J. Biomed. Opt. 15, 011109 (2010).

[4] D. Briers, D. D. Duncan, E. R. Hirst, S. J. Kirkpatrick, M. Larsson, W. Steenbergen, T. Stromberg, and O. B. Thompson, Laser speckle contrast imaging: Theoretical and practical limitations, J. Biomed. Opt. 18, 066018 (2013).

[5] B. Redding, M. A. Choma, and H. Cao, Speckle-free laser imaging using random laser illumination, Nat. Photonics 6 , 355 (2012).

[6] K. Kim, S. Bittner, Y. Zeng, S. F. Liew, Q. Wang, and H. Cao, Electrically pumped semiconductor laser with low spatial coherence and directional emission, Appl. Phys. Lett. 115, 071101 (2019).

[7] L. Xu, Y. Wang, Y. Jia, and W. Zheng, Low-coherence, high-power, high-directional electrically driven dumbbellshaped cavity semiconductor laser at $635 \mathrm{~nm}$, Opt. Lett. 45, 5097 (2020).

[8] R. Ma, Z. Wang, H. H. Zhang, W. L. Zhang, and Y. J. Rao, Imaging through opacity using a near-infrared low-spatialcoherence fiber light source, Opt. Lett. 45, 3816 (2020).

[9] Y. Gao, Y. Cui, L. Ji, D. Rao, X. Zhao, F. Li, D. Liu, W. Feng, L. Xia, J. Liu, H. Shi, P. Du, J. Liu, X. Li, T. Wang, T. Zhang, C. Shan, Y. Hua, W. Ma, X. Sun, X. Chen, X. Huang, J. Zhu, W. Pei, Z. Sui, and S. Fu, Development of low-coherence high-power laser drivers for inertial confinement fusion, Matter Radiat. Extremes 5, 065201 (2020).

[10] Y. Gao, L. Ji, X. Zhao, Y. Cui, D. Rao, W. Feng, L. Xia, D. Liu, T. Wang, H. Shi, F. Li, J. Liu, D. Pengyuan, X. Li, J. Liu, T. Zhang, C. Shan, Y. Hua, W. Ma, Z. Sui, J. Zhu, W. Pei, S. Fu, X. Sun, and X. Chen, High-power, lowcoherence laser driver facility, Opt. Lett. 45, 6839 (2020).

[11] Z. Zhong, B. Li, H. Xiong, J. Li, J. Qiu, L. Hao, and B. Zhang, Effective optical smoothing scheme to suppress laser plasma instabilities by time-dependent polarization rotation via pulse chirping, Opt. Express 29, 1304 (2021).

[12] Y. Huang, H. Chen, N. K. Fontaine, Y. Zhang, H. Huang, M. Mazur, J. C. Alvarado-Zacarias, R. Ryf, D. T. Neilson, G. Li, R. Amezcua-Correa, J. Carpenter, Y. Song, and M. Wang, Optical broadcasting employing incoherent and lowcoherence spatial modes for bi-directional optical wireless communications, J. Lightwave Technol. 39, 833 (2021). 
[13] R. Ma, J. Q. Li, J. Y. Guo, H. Wu, H. H. Zhang, B. Hu, Y. J. Rao, and W. L. Zhang, High-power low spatial coherence random fiber laser, Opt. Express 27, 8738 (2019).

[14] S. Knitter, C. Liu, B. Redding, M. K. Khokha, M. A. Choma, and H. Cao, Coherence switching of a degenerate VECSEL for multimodality imaging, Optica 3, 403 (2016).

[15] T. D. Visser, G. P. Agrawal, and P. W. Milonni, Fourier processing with partially coherent fields, Opt. Lett. 42, 4600 (2017).

[16] S. A. Wadood, B. E. Nussbaum, T. D. Visser, T. G. Brown, G. P. Agrawal, and A. N. Vamivakas, A Fourier processor for partially coherent fields, OSA Continuum 3, 2843 (2020).

[17] H. Cao, R. Chriki, S. Bittner, A. A. Friesem, and N. Davidson, Complex lasers with controllable coherence, Nat. Rev. Phys. 1, 156 (2019).

[18] M. Koivurova, T. Hakala, J. Turunen, A. T. Friberg, M. Ornigotti, and H. Caglayan, Metamaterials designed for enhanced ENZ properties, New J. Phys. 22, 093054 (2020).

[19] O. Svelto, Principles of Lasers (Springer, New York, 2010).

[20] A. Forbes, Laser Beam Propagation: Generation and Propagation of Customized Light (CRC Press, Boca Raton, 2014).

[21] See Supplemental Material at http://link.aps.org/ supplemental/10.1103/PhysRevLett.127.153902 for full derivation and technical details.

[22] A. G. Fox and T. Li, Resonant modes in a maser interferometer, Bell Syst. Tech. J. 40, 453 (1961).

[23] E. Wolf, New theory of partial coherence in the space-frequency domain. Part I: Spectra and cross spectra of steady-state sources, J. Opt. Soc. Am. 72, 343 (1982).

[24] E. Wolf and G. S. Agarwal, Coherence theory of laser resonator modes, J. Opt. Soc. Am. A 1, 541 (1984). 